\title{
Genetic diversity assessment of lily genotypes native to Korea based on simple sequence repeat markers
}

\author{
Shipra Kumari · Young-Sun Kim • Bashistha Kumar Kanth • Ji-Young Jang • Geung-Joo Lee
}

Received: 16 June 2019 / Revised: 6 August 2019 / Accepted: 7 August 2019

(c) Korean Society for Plant Biotechnology

\begin{abstract}
Molecular characterization of different genotypes reveals accurate information about the degree of genetic diversity that helps to develop a proper breeding program. In this study, a total of 30 EST-based simple sequence repeat (EST-SSR) markers derived from trumpet lily (Lilium longiflorum) were used across 11 native lily species for their genetic relationship. Among these 30 markers, 24 SSR markers that showed polymorphism were used for evaluation of diversity spectrum. The allelic number at per locus ranged from 1 at SSR2 locus to 34 alleles at SSR15 locus, with an average of 11.25 alleles across 24 loci observed. The polymorphic information content, PIC, values ranged from 0.0523 for SSR9 to 0.9919 for SSR2 in all 24 loci with an average of 0.3827 . The allelic frequency at every locus ranged from $0.81 \%$ at SSR2 locus to $99.6 \%$ at SSR14 locus. The pairwise genetic dissimilarity coefficient revealed the highest genetic distance with a value of $81.7 \%$ was in between $L$. dauricum and $L$. amabile. A relatively closer genetic distance was found between $L$. lancifolium and $L$. dauricum, L. maximowiczii and L. concolor, L. maximowiczii and L. distichum (Jeju), L. tsingtauense and L. callosum, $L$. cernuum and $L$. distichum (Jeju ecotype), of which dissimilarity coefficient was $50.0 \%$. The molecular fingerprinting based on microsatellite marker could serve boldly to recognize genetically distant accessions and to sort morphologically close as well as duplicate accessions.
\end{abstract}

Keywords EST-derived simple sequence repeats (EST-SSR),

${ }^{\dagger}$ This authors contributed equally to this work.

S. Kumari ${ }^{\dagger, \#} \cdot$ Y.-S. Kim ${ }^{\dagger}$ - B. K. Kanth - J.-Y. Jang •

G.-J. Lee $(\bowtie)$

Department of Horticultural Science, Chungnam National

University, Daejeon 34134, Korea

e-mail: gjlee@cnu.ac.kr

${ }^{\#}$ Current Address: Department of Agriculture Biotechnology, National Academy of Agriculture Science, Rural Development Administration, Jeonju, Jeollabuk-do 54874, Korea
Genetic diversity, Native lily, Polymorphic information content (PIC).

\section{Introduction}

The genus Lilium from family liliacea is widely dispersed in temperate areas like Europe, Asia, and North America (Liang et al. 2000; Patterson et al. 2002). Lily is a main flowering crop next to rose worldwide, in the market of cut flower. They have medicinal, food and economic value. Lilies are perennials herb with an abundant number of species and cultivars growing in open field and propagate asexually form underground scaly bulbs (Simpson 2011). Hybrid varieties have been generated making cross between Lilium species using conventional breeding as well as selections technique based on morphological characteristics like flower colors and shapes (Van Tuyl et al. 1991. Most of genetic diversity studies in Lilium are mainly dependent on morphological traits, but attempts were made in the diversity test for disease resistance such as Fusarium or Botrytis (Jang et al. 2015; Jang et al. 2018).

The cytological and morphological studies have been replaced by molecular studies like inter-simple sequence repeat, internal transcribed spacer (ITS) regions of rRNA genes and randomly amplified polymorphic DNA. Molecular marker studies in Lilium are not advanced compared to other plant species because of their large genome sizes. Molecular studies of wild Lilium species has been increasing and the reported molecular markers are ISSR (Xi et al. 2012), SRAP (Robarts and Wolfe 2014), RAPD (Huang et al. 2009), and ITS (Kumari et al. 2018; Sultana et al. 2011) to explore diversity of wild Lilium species. So, molecular techniques are required to systematically study of wild Lilium species in depth. Molecular markers related to resistance traits can indirectly decreased breeding and selection processes by MAS (marker assisted selection) 
especially in polygenic complex traits like lilies.

Genetic diversity analysis explains better variation degree between and within collected germplasm (Du et al. 2015; Biswas et al. 2018). Genetic diversity is a pre-condition for successful plant breeding (Du et al. 2015; Ulukan 2009). A lot of approaches have been applied in plant genetic diversity analysis which includes the use of morphological, biochemical, DNA-based markers and agronomical (Mohammadi and Prasanna 2003). But the selection of approaches are decided by objectives, required information and resources.

Out of various different molecular marker systems, satellite loci, also known like simple sequence repeat (SSR) has many benefits like high reproducibility, high polymorphism, high transferability and abundance over other systems (Biswas et al. 2018; Park et al. 2009). Molecular markers are potent tools to assess genetic variation and to elucidate genetic relationships among and within species. Simple sequence repeat (SSRs) is one of the most commonly used molecular markers. It is PCR-based marker that is not only sufficient across genome, but also cost-effective to be used. Compared with other markers, it is co-dominant, interspersed, abundant and highly reproducible all over the genome (Panaud et al. 1996; Temnykh et al. 2000).

The polymorphic SSR markers can be used efficiently to assist genetic grouping of lily genotypes and consequently lessen the timeline for development of lily cultivars. Therefore, the goal of this study was to establish the genetic relationship among native Korean lily genotypes that were collected in Korean peninsula, and ultimately select unique parents used for breeding program in Korea using SSR markers. This is the first study of its kind to report diversity analysis in the native Korean lilies using SSR markers, which will be greatly applicable when they are proved to be useful.

\section{Materials and Methods}

Plant materials

Eleven native lily species which were collected from different parts of Korea were used in this study. These include Lilium distichum Nakai (Martagon division; Malnari), $L$. lancifolium Thunb. (Asiatic division; Chamnari), Lilium dauricum Ker-Gawler (Asiatic division; Nalgae Haneulnari), L. leitlinii var. maximowiczii (Asiatic division; Joongnari), L. tsingtauense Gilg (Martagon division; Haneul Malnari), L. callosum Siebold \& Zucc (Asiatic division; Tdangnari), L. cernuum Komar. (Asiatic division; Solnari), L. concolor Salisb. (Asiatic division; Haneulnari), L. hansonii Leichtl.
(Martagon division; Seom Malnari), Lilium amabile Palibin (Asiatic division; Teoljoongnari), and $L$. distichum Nakai (Martagon; Jeju Malnari). Native lilies and their floral characteristics have been reported previously (Kumari et al. 2018).

\section{Isolation of genomic DNA}

Lily bulbs of each species were planted and germinated plants were grown in a green house at $25^{\circ} \mathrm{C} \pm 1{ }^{\circ} \mathrm{C}$ under natural conditions in a research farm of Chungnam National University. Genomic DNA was isolated from the leaves following CTAB method (Zheng et al. 1995). DNA samples are evaluated both qualitatively and quantitatively by Nanodrop Lite (Thermo Fisher Scientific Inc., U.S.A).

Selection of SSRs and amplification for genetic diversity evaluation

A total of thirty SSR primer pairs were choosen for genetic diversity analysis for eleven native lily species. Primers names, sequences and expected amplicon size were listed in Table 1. Primers indicating polymorphic bands were selected and those showing monomorphic banding patterns were not considered. Finally, twenty-four SSR markers were used for genotyping assays.

PCR amplification was performed in a cocktail of $20 \mu \mathrm{l}$ containing template DNA $30 \mathrm{ng}, 0.5 \mu \mathrm{M}$ of forward and reverse primer each, Taq 2X Master Mix (New England Biolabs Inc. London, UK) and nuclease free water. The PCR reactions were conducted in TaKaRa PCR Thermocycler Dice (Takara Bio Inc., Japan) with initial denaturation step at $95^{\circ} \mathrm{C}$ for $5 \mathrm{~min}, 35$ cycles of $95^{\circ} \mathrm{C}$ for $1 \mathrm{~min}$, for $1 \mathrm{~min}$ at $50^{\circ} \mathrm{C}$ to $65^{\circ} \mathrm{C}$ and $72^{\circ} \mathrm{C}$ for $1 \mathrm{~min}$; and final extension at $72^{\circ} \mathrm{C}$ for $7 \mathrm{~min}$.

The amplified SSR products were separated by $2.0 \%$ agarose gel electrophoresis. The DNA samples were electrophoresed using $0.5 \mathrm{X}$ TBE buffer $(\mathrm{pH} 8.3$ ) at $110 \mathrm{~V}$ for 20 40 min. on Mupid-2 Plus submarine electrophoresis system (Advance, Japan). The gel analysis was analyzed by GelAnalyzer (http://www.gelanalyzer.com /index.html). Markers having polymorphic information content (PIC) values in the range of 0.0523 to 0.991 were selected.

Data analysis

Based on the presence or absence of bands, amplicons were scored for every SSR primer pairs, and a binary data matrix of 1 and 0 was generated for each marker system. 
Table 1 List of thirty EST-SSR primers selected for PCR amplification and genetic diversity evaluation

\begin{tabular}{|c|c|c|c|c|}
\hline $\begin{array}{c}\text { SSR } \\
\text { marker }\end{array}$ & $\begin{array}{c}\text { Repeat } \\
\text { motif }\end{array}$ & Forward primer & Reverse primer & $\begin{array}{c}\text { Size of } \\
\text { amplified } \\
\text { fragment (bp) }\end{array}$ \\
\hline SSR1 & $(\mathrm{CAC}) 5$ & AАССТАСАСТТСССТСТТСТТТ & TTATTAGCAGCAACATTCAACT & $500 / 384$ \\
\hline SSR2 & $(\mathrm{CCA}) 5$ & GTCTCACAGCCCTCCTACAC & ACTTTTCTTCGAGAATCAAGTG & 280 \\
\hline SSR3 & (GA)9 & AACTCCACAATAAGAGGGAAG & TGTTGTACTTGGCTGTTACATT & 292 \\
\hline SSR4 & $(\mathrm{CAG}) 6$ & CAATCCTCTGTGTCAATAACTG & GTAACAACCGGATCTTTAACTC & 187 \\
\hline SSR5 & $(\mathrm{CCA}) 5$ & ACAGCCCTCCTACACAACTC & GTCATAAACGGGTAGGGTTT & 120 \\
\hline SSR6 & $(\mathrm{GGA}) 9$ & CCAACAATTTTGATTACATGG & ATTCAAGCAATATCTCATCCTC & 213 \\
\hline SSR7 & $(\mathrm{CAA}) 4$ & CCTACATGTGCATCTCAAATAC & TAACAGATCCAGCAAAGATATG & $320 / 230$ \\
\hline SSR8 & $(\mathrm{CTT}) 4$ & CTGAAGCAAACCTAATTCCTAC & GATATGATAAAGGGCAAGACTC & 300 \\
\hline SSR9 & (ATC) 5 & CGGTAGTCTTAAGCAAGAAGTT & ACTGATATGGAGTTGGATGAGT & 303 \\
\hline SSR10 & $(\mathrm{CAC}) 9$ & ACTGGGGAGAATATCAAGAAC & AAAAACCAACTACAACACATCA & 288 \\
\hline SSR11 & (TCC)6 & CCACAATAAACGATGATGTCT & TAAGCATCATATCAAGCATAGC & 400 \\
\hline SSR12 & $(\mathrm{GCC}) 5$ & GATTGCACTCTATCAGTCACAG & TAATCCCTTTATGAAGATGGTC & 240 \\
\hline SSR13 & $(\mathrm{CT}) 7$ & CTATTTCССCTCCTTTGACC & AGATGGTGTCTGTTGAAGTTTT & 160 \\
\hline SSR14 & $(\mathrm{CT}) 8$ & CAGAGATACAAAGCAAAAACAA & AAGAGTGGAGGATCTGAAGAG & 154 \\
\hline SSR15 & (CTC)4 & TTTCTCGGTTGGCCCCTATG & AGATGAGACATTGCCGGCTG & $350 / 300$ \\
\hline SSR16 & $(\mathrm{AAT}) 4$ & CTGATCTGGTAGACGAGCACGA & AGATGCTCACAAACACCGTCAA & 220 \\
\hline SSR17 & (TAA)6 & TGCGCTCTGTAGTGTGTTCCAT & CAGACATGCCATGAAAACGAAG & $225 / 215$ \\
\hline SSR18 & (TGG)4 & CCCGTCAAGCAAGGATATCAAG & ССТTCTCTTCCTTCTTCGGCTC & 400 \\
\hline SSR19 & $(\mathrm{GGC}) 5$ & TGACTTCCGCAGAGATAGAGGC & CTCATGTCAGTCCCATGCACTC & $210 / 200$ \\
\hline SSR20 & (GCA)4 & AAGCATGCTGAGCTGTTGTCAG & CTGCTTGAGTTGGTGTTGTTCG & 160 \\
\hline SSR21 & (GTG)4 & TGGTGGTAGAGGGCAATCATCT & CTTGAGCAAAACAGACATCCCC & 400 \\
\hline SSR22 & (CTT)4 & TCCCAATGAAGAACACCСТCTC & GACCTGGAAGAAGTCGGTGATG & $200 / 190$ \\
\hline SSR23 & $(\mathrm{CCT}) 4$ & GAACCGGTCTTCTTCCCTCAAC & GCCTCTCCACTGCAACCAGTAA & 160 \\
\hline SSR24 & (CTC)4 & CAAAGGAGAAGCGATGAGTCGT & GGAACCATCGGTGAGAAGAGTG & 220 \\
\hline SSR25 & $(\mathrm{GAG}) 4$ & AGTCAGATGCAGGAGAGGATGG & GTCCTCCGCTTCCACAAGTTC & 300 \\
\hline SSR26 & $(\mathrm{GCG}) 4$ & CGAAATTAGGGTTAGGGTTCCG & GTCGGAGAAATTGCTCGAATTG & 210 \\
\hline SSR27 & $(\mathrm{GCG}) 4$ & CAGGAGCTTAGGTGCTGCTGTT & TAGTGCTGCTCAGTTGTGTGGG & 260 \\
\hline SSR28 & (TGC)4 & TACATCTGCTGGGTCCATCCTT & TGACAGCATTGTGAATGGAAGC & 200 \\
\hline SSR29 & $(\mathrm{CGC}) 4$ & TTCCATTTCTAAACCCACACCG & TGATTTAGCTTTCAGCGCAGTG & 155 \\
\hline SSR30 & $(\mathrm{GCC}) 4$ & CCCTTTGATGAAGCAGAAGTGC & TTGCACAGAAAATCACGATGCT & 155 \\
\hline
\end{tabular}

Microsatellite loci repeats were assayed based on the detected number of alleles observed with the PCR amplicon profile. Major allele frequency, PIC values were computed for each and every SSR locus using equation - PIC $=1-\sum \mathrm{Pi}$, where $p$ represents frequency of $i^{\text {th }}$ allele (Shete et al. 2000). GenAIEx 6.5 was used to calculate diversity (Peakall and Smouse 2012). The binary data matrix was then analyzed by the NTSYSpc statistical package version 2.2 (Rohif 2002). The genetic similarity based on Jaccard's similarity coefficients was calculated using the matrix data. The relationships among eleven lily genotypes was displayed by two dendograms constructed using the Unweighted Pair Group Method with Arithmetic Mean (UPGMA). The Pearson's correlation between similarity coefficients were determined from data among all eleven genotypes.

\section{Results and Discussion}

Allele spectrum for SSR polymorphism among native lily

All 30 SSR primer sets selected for amplification produced PCR amplicon and 6 primer sets produced monomorphic amplicons. Thus, a total of 24 primer sets producing high polymorphic bands were used to analyze the genetic relationship and diversity among eleven lily genotypes.

24 high polymorphic SSRs were selected for the genetic relationship analysis of 11 native lilies (Table 1). The clustering pattern exhibited that the native lilies formed distinct group in phylogenetic dendrogram. The degree of polymorphism among lily genotype was appraised by calculating the number of alleles and PIC values for every of the thirty SSR loci analyzed. A total of 342 alleles were observed at the loci of thirty microsatellite markers across eleven lily genotypes. 
Table 2 List of 24 SSR markers with their allele frequency, number of alleles, diversity and PIC values found among 11 native lily genotypes

\begin{tabular}{|c|c|c|c|c|c|}
\hline SSR Marker & Repeat motif & No. of alleles & Allele frequency & Genetic diversity & PIC value \\
\hline SSR 1 & $(\mathrm{CAC}) 5$ & 8 & 0.8586 & 0.2975 & 0.1414 \\
\hline SSR 2 & $(\mathrm{CCA}) 5$ & 1 & 0.0081 & 0.1652 & 0.9919 \\
\hline SSR 3 & (GA)9 & 3 & 0.8262 & 0.1652 & 0.1738 \\
\hline SSR4 & $(\mathrm{CAG}) 6$ & 4 & 0.486 & 0.4958 & 0.514 \\
\hline SSR5 & $(\mathrm{CCA}) 5$ & 8 & 0.62 & 0.4628 & 0.38 \\
\hline SSR6 & (GGA)9 & 2 & 0.5508 & 0.3966 & 0.4492 \\
\hline SSR7 & $(\mathrm{CAA}) 4$ & 7 & 0.3969 & 0.4958 & 0.6031 \\
\hline SSR 8 & $(\mathrm{CTT}) 4$ & 11 & 0.8424 & 0.4958 & 0.1576 \\
\hline SSR9 & (ATC)5 & 7 & 0.9477 & 0.2975 & 0.0523 \\
\hline SSR11 & (TCC)6 & 6 & 0.2025 & 0.1652 & 0.7975 \\
\hline SSR12 & $(\mathrm{GCC}) 5$ & 2 & 0.5265 & 0.4958 & 0.4735 \\
\hline SSR14 & $(\mathrm{CT}) 8$ & 10 & 0.9963 & 0.3966 & 0.1171 \\
\hline SSR 15 & (CTC) 4 & 34 & 0.7452 & 0.2975 & 0.2548 \\
\hline SSR16 & $(\mathrm{AAT}) 4$ & 31 & 0.891 & 0.1652 & 0.109 \\
\hline SSR17 & (TAA) 6 & 15 & 0.5022 & 0.1652 & 0.4978 \\
\hline SSR18 & (TGG)4 & 23 & 0.5184 & 0.2975 & 0.4816 \\
\hline SSR19 & $(\mathrm{GGC}) 5$ & 20 & 0.9153 & 0.3966 & 0.0847 \\
\hline SSR20 & $(\mathrm{GCA}) 4$ & 13 & 0.3726 & 0.3966 & 0.6274 \\
\hline SSR21 & (GTG)4 & 12 & 0.6561 & 0.2975 & 0.3439 \\
\hline SSR22 & $(\mathrm{CTT}) 4$ & 7 & 0.8829 & 0.2975 & 0.1171 \\
\hline SSR23 & $(\mathrm{CCT}) 4$ & 13 & 0.5346 & 0.4958 & 0.4654 \\
\hline SSR25 & (GAG)4 & 5 & 0.7614 & 0.2975 & 0.2386 \\
\hline SSR26 & (GCG)4 & 22 & 0.729 & 0.3966 & 0.271 \\
\hline SSR29 & (CGC)4 & 6 & 0.8424 & 0.3966 & 0.1576 \\
\hline Mean & & 11.25 & 0.6505 & 0.3429 & 0.3827 \\
\hline
\end{tabular}

The results showed that most of the primers produced distinct polymorphisms among the genotypes studied demonstrating the strong nature of microsatellites in informative polymorphism. Among the polymorphic markers, 2 produced two alleles each, 2 produced six alleles each, 3 generated seven alleles each, 2 produced 8 alleles each, 2 produces 13 alleles each, and only one produced 34 (maximum) along with other marker produced alleles varying from 10 to 31 (Table 3). The range of allelic number per locus is from 1 to 34 alleles with an average of 11.25 alleles across the 24 loci. The highest allelic number (34.0) detected in the locus SSR15 and the lowest alleles (1) were observed on SSR2.

The amplicon size in all 11 genotypes for each marker allele varied from 200-100 bp. produced by SSR6 and 3000-274 bp. produced by SSR15. Out of 342 alleles scored, all of 270 were established to be polymorphic. Maximum polymorphic allelic bands (34) were detected with the marker SSR15, while the minimum polymorphic allelic band (1) was obtained from SSR1.

Figure 1 represents an agarose gel image of amplified fragments obtained by SSR15 \& SSR16. A wide spectrum of produced 34 alleles was found with SSR15 locus, followed by 31 alleles in SSR16 locus. This demonstrates that these markers might be potentially employed for molecular characterization of native lilies from various sources. However, some markers produced only few alleles like SSR2 gave only one, SSR6 \& SSR12 each gave only two. Three markers were robust enough to discriminate specifically various genotypes or incompatible accessions of the same genotype.

Evaluation of Genetic diversity based on PIC value

SSR markers are very high informative polymorphic like evident from its polymorphism information content PIC value. PIC value reflects allele diversity and frequency among varieties. The PIC value is a calculation of polymorphism among varieties for a marker locus applied in the analysis of linkage. The PIC value of every marker, which may be estimated based on its alleles, varied greatly for all evaluated SSR loci from 0.0523 to 0.9919 with an 
Table 3 Pairwise genetic distance indices (Jaccard coefficient) explaining dissimilarity among 11 native lilies obtained from microsatellite marker analysis

\begin{tabular}{|c|c|c|c|c|c|c|c|c|c|c|c|}
\hline & L. distichum I & lancifolium $L$ & danricum & L. maximowiczii $L$. & tsingtauense $L$ & callosum & L. cernumm & L. concolor & L. hansonii & L. amabile & $\begin{array}{l}\text { L. distichum } \\
\text { (Jeju) }\end{array}$ \\
\hline L. distichum & 0.0000 & & & & & & & & & & \\
\hline L. lancifolium & 0.4564 & 0.0000 & & & & & & & & & \\
\hline L. dauricum & 0.6124 & 0.5000 & 0.0000 & & & & & & & & \\
\hline L. maximowiczii & 0.6455 & 0.6770 & 0.6770 & 0.0000 & & & & & & & \\
\hline L. tsingtauense & 0.6770 & 0.7071 & 0.7071 & 0.4564 & 0.0000 & & & & & & \\
\hline L. callosum & 0.6770 & 0.6455 & 0.6455 & 0.4564 & 0.5000 & 0.0000 & & & & & \\
\hline L. cernuит & 0.5774 & 0.5401 & 0.5401 & 0.5774 & 0.6124 & 0.5401 & 0.0000 & & & & \\
\hline L. concolor & 0.7071 & 0.6124 & 0.6770 & 0.5000 & 0.6770 & 0.5401 & 0.7071 & 0.0000 & & & \\
\hline L. hansonii & 0.6770 & 0.7071 & 0.7638 & 0.4564 & 0.5774 & 0.5774 & 0.6124 & 0.5401 & 0.0000 & & \\
\hline L. amabile & 0.6124 & 0.7071 & 0.8165 & 0.6124 & 0.7071 & 0.6455 & 0.6770 & 0.6124 & 0.5774 & 0.0000 & \\
\hline L. distichum (Jeju) & 0.5774 & 0.6124 & 0.7360 & 0.5000 & 0.6124 & 0.6124 & 0.5000 & 0.5774 & 0.4564 & 0.5401 & 0.0000 \\
\hline
\end{tabular}
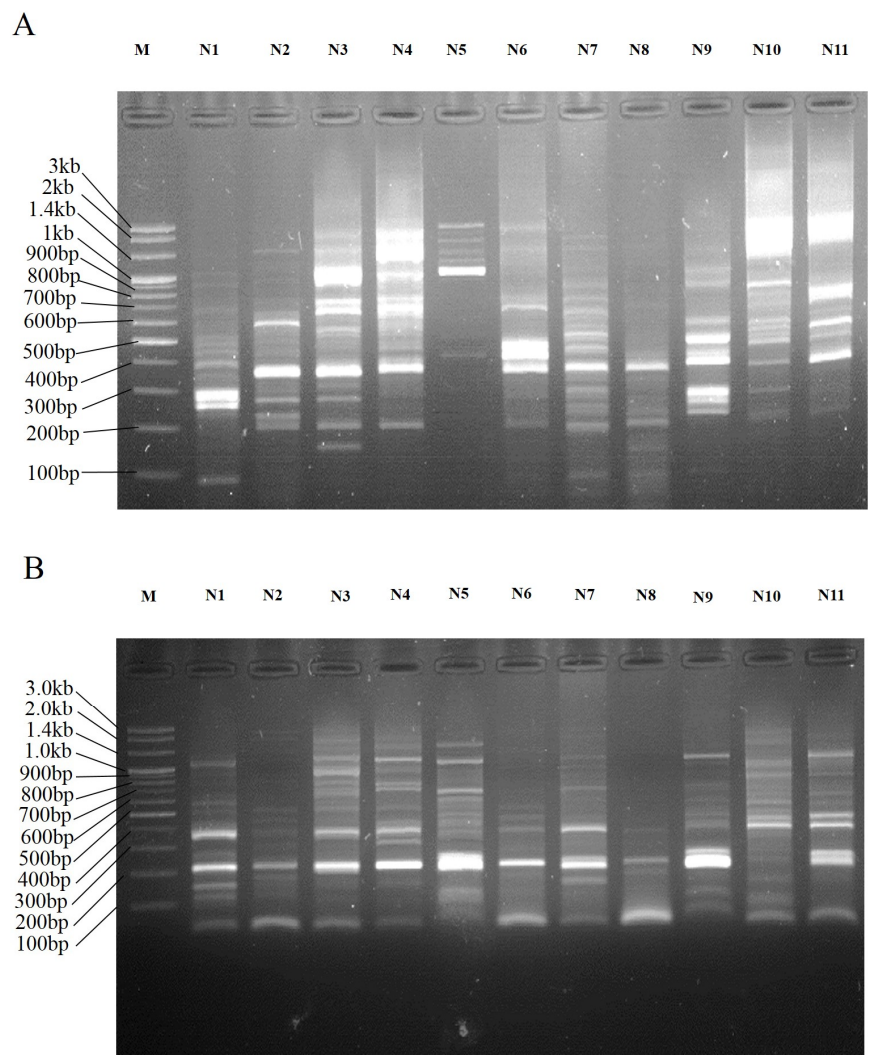

Fig. $12 \%$ Agarose electrophoresis showing amplification profile of EST-SSR markers in eleven native lilies with SSR15 (A) and SSR 16 (B) markers. N1- Lilium distichum, N2- Lilium lancifolium, N3- Lilium dauricum, N4- Lilium maximowiczii, N5- Lilium tsingtauense, N6- Lilium callosum, N7- Lilium cernuum, N8- Lilium concolor, N9- Lilium hansonii, N10- Lilium amabile and N11L. distichum (Jeju). M- $3.0 \mathrm{~Kb}$ Ladder

average of 0.3827 (Table 3). The high PIC value 0.9919 was obtained for SSR2, followed by respectively SSR8 (0.8424), SSR11 (0.7975) and SSR20 (0.6274). The PIC values of 24 markers from 0.0523 to 0.9919 with an average of 0.3827 which are lower than Lilium genus (Lee et al. 2011), groundnut (Cuc et al. 2008), Cassava (Moyib et al. 2007), and maize (Enoki et al. 2002).
Species diversity based on genetic clustering using genetic distance indices

SSR genetic distance applies to the genetic variance among populations, which may be calculated by a variety of parameters in connection with frequency of a particular trait. By using the binary data that was obtained from sample DNA profile, UPGMA-based dendrogram was con- 


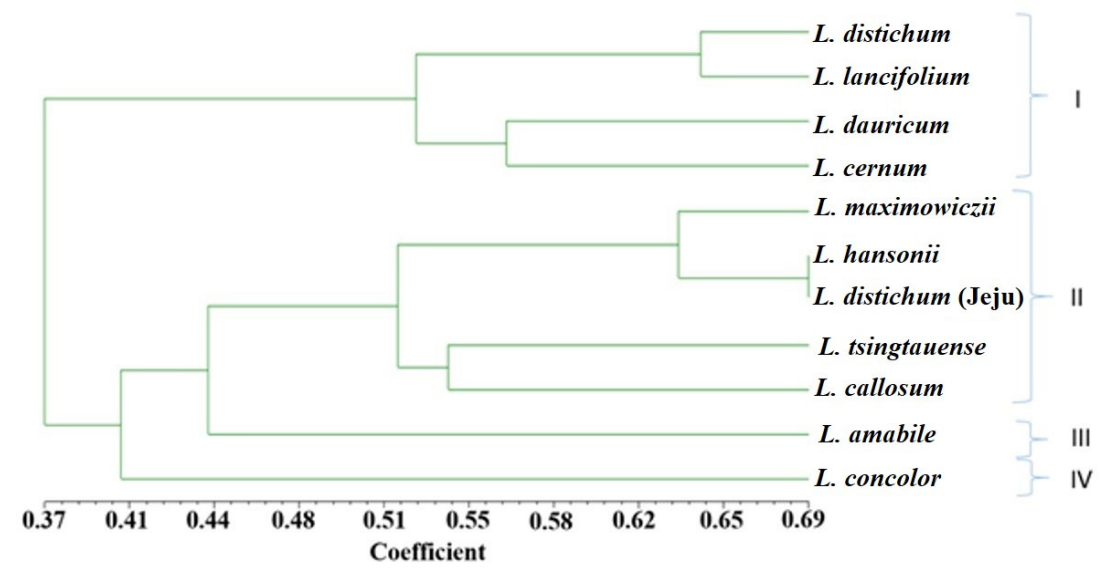

Fig. 2 A UPGMA clustering dendrogram representing the genetic relationships among eleven native lilies on the alleles detected by twenty-four microsatellite markers

structed. Genotypes that are genetically derived from similar types are clustered together.

Using 63\% similarity like the threshold for UPGMA clustering, we obtained four major genetic clusters (Fig. 2). Cluster I was the second biggest group containing four genotypes- $L$. distichum, $L$. lancifolium, $L$. dauricum and L. cernuum. This group contained two sub-clusters, a subgroup of $L$. distichum and L. lancifolium, and another subgroup of L. dauricum and L. cernuum. The subgroup of $L$. distichum and L. lancifolium showed $45.64 \%$ dissimilarity, whereas $L$. dauricum and $L$. cernuum in another group showed $54.01 \%$ dissimilarity Table 3 .

Cluster II was the biggest group which contained four genotypes - L. leichtlinii, L. hansonii,, L. distichum (Jeju), L. tsingtauense and L. callosum. Cluster II contained two sub cluster, one of $L$. tsingtanense \& L. distichum (Jeju) which showed similarity $47.06 \%$, second of $L$. maximowiczii, $L$. hansonii and L. distichum (Jeju). L. hansonii and $L$. distichum (Jeju) showed $45.64 \%$ dissimilarity. Cluster III and Cluster IV contained only lily genotype - L. amabile and $L$. concolor respectively.

Pairwise genetic dissimilarity

A dissimilarity matrix determines the relationship among various genotypes. The pairwise genetic dissimilarity indices (Table 3) suggested that the highest genetic dissimilarity was between $L$. dauricum \& L. amabile with $81.65 \%$ ); $L$. dauricum \& L.hansonii with $76.38 \%$; L. dauricum \& L. distichum (Jeju) with $73.60 \%$; L. distichum \& L. concolor, L. lancifolium \& L. tsingtanense, L. lancifolium \& L. hansonii, L. lancifolium \& L. amabile, $L$. dauricum \& $L$. tsingtauense, L. tsingtauense \& L. amabile, L. cernum \& L. concolor with $70.71 \%$. The lowest genetic dissimilarity was found among lilies was between $L$. lancifolium \& $L$. lancifolium, L. maximowiczii \& L. tsingtauense, L. maximowiczii \& L.callosum, L. maximowiczii \& L. hansonii, $L$. hansonii \& L. distichum (Jeju) which showed 45.64\%, followed by $L$. lancifolium \& L. dauricum, L. maximowiczii \& L. concolor, L. maximowiczii \& L. distichum (Jeju), L. tsingtauense \& L. callosum, L. cernuum \& L. distichum (Jeju) which demonstrated average of $50.00 \%$. In this way, SSR markers, which bear enough power of resolution, can be used to perceive the difference between genotypes. SSR markers are being used as a powerful tool to identify and to characterize genetically distant cultivars from various sources.

In conclusion, the study indicated that the lily SSR markers are robust molecular markers and are neutral, co-dominant and could be a potent tool to assess cultivarsgenetic variability. The information about the genetic diversity will be very useful to properly identify and select appropriate parents for breeding programs. The other SSR or SNP markers produced from the transcriptome evaluation among lily populations in our group will be further evaluated for genetic map construction or exploring SSR or SNP loci which are inherited with traits of interest.

\section{Acknowledgement}

This research was supported by the academic research fund of Chungnam National University.

\section{References}

Biswas MK, Nath UK, Howlader J, Bagchi M, Natarajan S, Kayum 
MA, Kim HT, Park JI, Kang JG, Nou IS (2018) Exploration and exploitation of novel SSR markers for candidate transcription factor genes in Lilium species. Genes 9:97

Cuc LM, Mace ES, Crouch JH, Quang VD, Long TD, Varshney RK (2008) Isolation and characterization of novel microsatellite markers and their application for diversity assessment in cultivated groundnut (Arachis hypogea). BMC Plant Biol $8: 55$

Du F, Jiang J, Jia H, Zhao XY, Wang WH, Gao QK, Mao WH, Wu Y, Zhang L, Griersone D, YP Xia, Gao ZS (2015). Selection of generally applicable ssr markers for evaluation of genetic diversity and identity in Lilium. Biochem Syst Ecol 61: 278-285

Enoki H, Sato H, Koinuma K (2002) SSR analysis of genetic diversity among maize inbred lines adapted to cold regions of Japan. Theor Appl Genet 104:1270-1277

Huang YF, Yang MX, Zhang H, Zhuang XY, Wu XH, Xie W (2009) Genetic diversity and genetic structure analysis of the natural populations of Lilium brownii from Guangdong, China. Biochem Genet 47: 503e-510

Kumari S, Kanth BK, Jeon Y, Jang J-Y, Kim H-S, Lee G-J (2018) Internal transcribed spacer-based CAPS marker development for Lilium hansonii identification from wild Lilium native to Korea. Sci Horticulturae 236:52-59

Lee SI, Park KC, Song YS, Son JH, Kwon SJ, Na JK, Kim JH, Kim NS (2011) Development of expressed sequence tag derivedsimple sequence repeats in the genus Lilium. Genes Genomics 33: $727-733$

Liang SY, Tamura M (2000) Lilium L.. In: Wu ZY \& Raven PH (eds.) Flora of China 24. Science Press, Beijing; \& Missouri Botanical Garden Press, St. Louis, pp 135-159

Mohammadi S, Prasanna B (2003) Analysis of genetic diversity in crop plants - salient statistical tools and considerations Crop Sci 43: $1235-1248$

Moyib OK, Odunola OA, Dixon AGO (2007) SSR markers reveal genetic variation between improved cassava cultivars and landraces within a collection of Nigerian cassava germplasm. Afric J Biotechnol 8: 2666-2674

Panaud O, Chenayd X, McCouch SR (1996) Development of microsatellite markers and characterization of simple sequence length polymorphism (SSLP) in rice (Oryza sativa L.). Mol General Genet 252: 597-607

Park YJ, Lee JK, Kim NS (2009) Simple sequence repeat polymorphisms (SSRPs) for evaluation of molecular diversity and germplasm classification of minor crops. Molecules 14:
4546-4569

Peakall R, Smouse PE (2012) GenAlEx 6.5: Genetic analysis in Excel. Population genetic software for teaching and researchan update. Bioinformatics 28: 2537-2539

Patterson TB, Givnish TJ (2002) Phylogeny, concerted convergence, and phylogenetic niche conservatism in the core Liliales: insights from rbcL and $\mathrm{ndhF}$ sequence data. Evolution. 56: 233-252. doi:10.1111/j.0014-3820.2002.tb01334.x.

Robarts DW, Wolfe AD (2014) Sequence-related amplified polymorphism (SRAP) markers: A potential resource for studies in plant molecular biology. Appl Plant Sci 2:1400017

Rohif FJ (2002) NTSYS-pc: Numerical taxonomy and multivariate analysis system (Ed. 2.2). Department of Ecology and Evolution, State University of NY, Stony Brook

Shete S, Tiwari H, Elston RC (2000) On estimating the heterozygosity and polymorphism information content value. Theor Pop. Biol 57: 265-271

Simpson, MG (2011). Plant Systematics. Academic Press. ISBN 978-0-08-051404-8

Sultana S, Lim YP, Bang JW, Choi HW (2011) Internal transcribed spacer (ITS) and genetic variations in Lilium native to Korea. Hortic Environ Biotechnol 52:502e-510

Temnykh S, Park WD, Ayres N, Cartinhour S, Hauck N, Lipovich L , Cho YG, Ishii T, McCouch SR (2000) Mapping and genome organization of microsatellite sequence in rice (Oryza sativa L.). Theor Appl Genet 100:697-712

Ulukan H (2009) The evolution of cultivated plant species: classical plant breeding versus genetic engineering. Plant Syst Evol 280:133-142

Van Tuyl JM, Straathof TP, Bino RJ, Kwakkenbos AAM (1988) Effect of three pollination methods on embryo development and seed set in intra- and interspecific crosses between seven Lilium species. Sex Plant Reprod 1:119-123

Van Tuyl JM, Van Dien MP, Van Creij MGM, Van Kleinwee TCM, Franken J, Bino RJ (1991) Application of in vitro pollination, ovary culture, ovule culture, and embryo rescue for overcoming incongruity barriers in interspecific Lilium crosses. Plant Sci 74:115-126

Xi ML, Sun LN, Qiu S, Liu JJ, Xu J, Shi JS (2012) In vitro mutagenesis and identification of mutants via ISSR in lily (Lilium longiflorum). Plant Cell Rep 31: 1043e-1048

Zheng K, Huang N, Bennett J, Khush GS (1995) PCR assisted marker based selection in rice breeding. IRRI Discussion Paper Series No. 12. IRRI. Manila. Philipines 5-6 